\title{
Una aproximación a la comercialización de la producción de los concentrados mineros
}

\author{
Ysaac Ospino Edery
}

\section{RESUMEN}

El presente artículo desarrolla un particular análisis sobre la implicancia de la comercialización de la producción de concentrados mineros para el periodo de 1989 hasta el 2007. Los volúmenes embarcados (exportados) de concentrados mineros en los últimos 10 años representan, en flete pagado a las empresas navieras extranjeras, montos equivalentes a los requeridos para poner en marcha una o dos refinerías, así como los pagos realizados a refinerías en el exterior por refinar esos concentrados.

Con la aplicación en extremo, de políticas económicas de promoción a las inversiones minera de carácter liberal, no se tiene un control - fiscalización de la valorización de la producción minero metalúrgico, lo cual estaría permitiendo la práctica de sub valuación o sobre valorización (transferencias).

Palabras clave: Producción de concentrados mineros, exportación de concentrados, flete marítimo, refinado, maquila de refinación, sobre valorización, sub valuación, transferencias de precios. 


\title{
Ysaac Ospino Edery
}

\begin{abstract}
The present article develops a particular analysis on the implication of the commercialization of the production of concentrated minerals for the period of 1989 until the 2007. The volumes embarked (exported) of concentrated minerals in the last 10 years, represent in paid load, the shipping companies foreign amounts equivalent to the required ones to start up one or two refineries, as well as the payments made to refineries in the outside to refine those concentrated.

With the application in end, of economic policies of promotion to the investments mining of liberal character, a control/fiscalización to the valuation of the metallurgical mining production is not had which would be allowing the practice of subestimate or on valuation (transference of prices).
\end{abstract}

Keywords: Concentrate mimihp production; exportation of concentrates; Maritime Treight; Rejimed; Trandference of prices.

\section{INTERROGANTES A RESOLVER}

En nuestro artículo "Una aproximación a los mecanismos para la determinación de los precios de los metales"1; nos referimos a la comercialización de la producción minero metalúrgica, como la razón del negocio de la inversión minera.

Es conocido el aporte de la actividad minera a la economía de nuestro país, el cual contribuye con alrededor del $60 \%$ del valor total exportado (Cuadro N. ${ }^{\circ} 1$ ), lo que también señala cuan dependiente y vulnerable es la misma con respecto al mercado internacional dada nuestra estructura primario exportadora (Gráfico N. ${ }^{\circ} 1$ ), aspecto que debe ser de preocupación constante a fin de potenciar otros sectores económicos con la finalidad de restarle preponderancia a esa dependencia.

La contribución del sector minero a las exportaciones se da, principalmente, por la venta de la producción en forma de concentrados, tal como se puede apreciar en el Cuadro $\mathrm{N} .{ }^{\circ} 2$, donde los más representativos son los concentrados de Zinc y concentrados de Cobre.

1. Ospino E., Ysaac. Pensamiento Critico. N. ${ }^{\circ}$; ; enero de 2008. Revista del Instituto de Investigaciones Económicas de la Universidad Nacional Mayor de San Marcos. 
El concentrado minero es producto de una etapa intermedia de valor agregado, completándose esta con la refinación como etapa final previa al inicio de los procesos de industrialización del metal.

Cuadro N. ${ }^{\circ}$ 1. De exportaciones \%.

\section{6}

$\begin{array}{lrrrrrrrrrrr}\text { Pesqueros } & 15.5 & 16.5 & 7.1 & 9.9 & 13.7 & 13.2 & 11.6 & 9.0 & 8.6 & 7.5 & 5.6 \\ \text { Agrícolas } & 5.1 & 6.9 & 5.6 & 4.6 & 3.6 & 3.0 & 2.8 & 2.5 & 2.5 & 1.9 & 2.4 \\ \text { Mineros } & 45.2 & 40.0 & 47.7 & 49.4 & 46.3 & 45.6 & 49.4 & 51.6 & 55.6 & 56.3 & 61.8 \\ \text { Petr y Deriv } & 6.0 & 5.5 & 4.0 & 4.1 & 5.5 & 5.6 & 6.8 & 6.8 & 5.0 & 8.8 & 7.4\end{array}$

$\begin{array}{llllllllllll}\text { No Tradici } & 27.0 & 30 & 34.2 & 30.8 & 29.4 & 31.1 & 29.2 & 28.8 & 27.2 & 24.7 & 22.1\end{array}$

$\begin{array}{llllllllllll}\text { Otros } & 1.2 & 1.1 & 1.4 & 1.2 & 1.5 & 1.5 & 1.2 & 1.3 & 1.1 & 0.8 & 0.7\end{array}$

$\begin{array}{lllllllllllll}\text { Total } & 100.0 & 100.0 & 100.0 & 100.0 & 100.0 & 100.0 & 100.0 & 100.0 & 100.0 & 100.0 & 100.0\end{array}$

Fuente: BCRP, SUNAT, y empresas.

Del 2005 al 2007, se registró un aumento excepcional por ingreso de exportaciones de la producción minero metalúrgica, lo cual se atribuye a la mejora sustantiva de los precios de los metales a escala internacional, lo que ha inducido, probablemente, a la realización de producciones marginales, lográndose un incremento de los volúmenes comercializados.

Gráfico N. ${ }^{\circ} 1$.

Perú: Evolución de las exportaciones (Millones de USFOB)

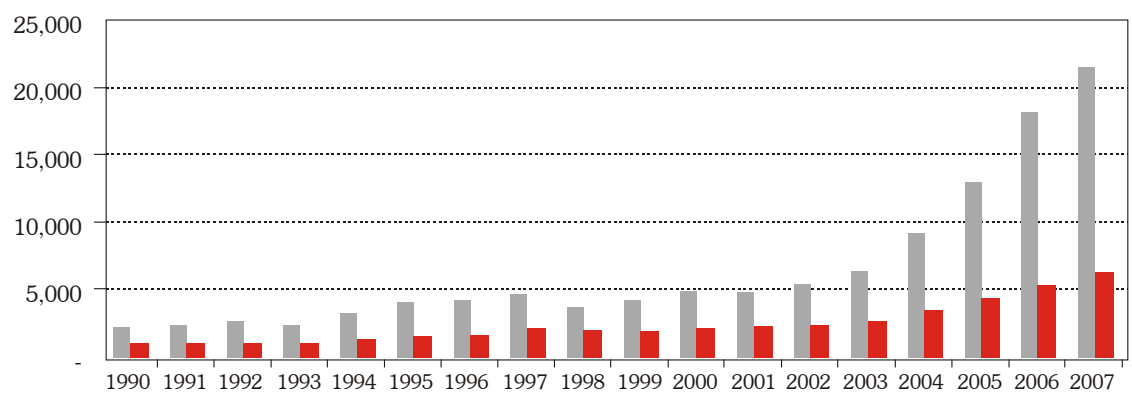

Productos primarios

Productos facturados 


\section{Ysaac Ospino Edery}

Cuadro N. ${ }^{\circ}$ 2. Perú: Exportaciones de Concentrados - (Miles de TMF/TMS)

\begin{tabular}{rrrrr}
\hline \multirow{2}{*}{ Año } & \multicolumn{2}{c}{ Concentrados de Zinc } & \multicolumn{2}{c}{ Concentrados de Cobre } \\
& \multicolumn{1}{c}{ TMF } & TMS e & TMF & TMS e \\
\hline 1989 & 388.2 & 705.8 & 38.3 & 109.4 \\
1990 & 462.0 & 840.0 & 43.3 & 123.7 \\
1991 & 345.8 & 628.7 & 34.1 & 97.4 \\
1992 & 369.0 & 670.9 & 40.3 & 115.1 \\
1993 & 438.5 & 797.2 & 28.8 & 82.2 \\
1994 & 442.3 & 804.1 & 46.0 & 131.4 \\
1995 & 426.5 & 774.5 & 62.9 & 179.7 \\
1996 & 477.0 & 867.2 & 69.0 & 197.1 \\
1997 & 544.2 & 989.4 & 85.3 & 243.7 \\
1998 & 535.0 & 972.7 & 63.8 & 182.2 \\
1999 & 545.0 & 990.9 & 86.5 & 247.1 \\
2000 & 667.0 & 1212.7 & 94.9 & 271.1 \\
2001 & 801.6 & 1457.4 & 233.0 & 637.0 \\
2002 & 1015.2 & 1845.8 & 366.9 & 1048.0 \\
2003 & 1065.6 & 1937.4 & 303.1 & 866.0 \\
2004 & 943.9 & 1716.1 & 465.3 & 1329.4 \\
2005 & 1001.7 & 1821.2 & 465.6 & 1328.5 \\
2006 & 988.3 & 1796.3 & 517.7 & 1479.1 \\
2007 & 1208.7 & 2197.6 & 762.4 & 2178.2 \\
\hline
\end{tabular}

Fuente: SUNAD/SUNAT/ Elaboración propia. / (e). Estimado

Cuadro N. ${ }^{\circ}$ 3. Exportaciones de Concentrados - Destino Geográfico (\%)

\begin{tabular}{lrrrrrr}
\hline & 2002 & $\%$ & 2003 & $\%$ & 2004 & $\%$ \\
\hline Europa & 505 & 27.5 & 661 & 33.9 & 730 & 38.7 \\
Norteamérica & 227 & 12.3 & 247 & 12.7 & 388 & 20.5 \\
Sud América (Bra) & 188 & 10.2 & 208 & 10.6 & 290 & 15.4 \\
Asia & 869 & 47.3 & 725 & 37.2 & 415 & 22.0 \\
Australia & 50 & 2.7 & 110 & 5.6 & 63 & 3.4 \\
Total & 1839 & 100 & 1951 & 100 & 1886 & 100 \\
\hline
\end{tabular}

Fuente: ILZSG/LATIZA

Aquí preguntamos: ¿Por qué no se exporta más volúmenes de refinados?, ¿por qué solo hemos podido poner en marcha (operaciones), dos refinerías en los últimos 40 años? (ambas construidas durante el periodo de gobierno de la Junta Militar 1968/1980). 


\section{Una aproximación a la comercialización de la producción de los concentrados mineros}

La primera, la de cobre en Ilo, administrada por la empresa estatal Minero Perú, hasta principios de la década de los 90 , construida con el objeto de refinar el cobre blister de la fundición de la Southern en Ilo, cercana a la refinería; blister procedente de los concentrados de cobre de sus minas Toquepala y Cuajone, empresa a la que se le cobraba los derechos de refinación (cargos por refinación) correspondiente, además de otros pagos por otros contenidos finos recuperables, así como de servir de indicador y regulador de las diferentes variables que intervienen en la valorización de un concentrado que luego es refinado, variables que se aprecian en la tabla $\mathrm{N} .^{\circ} 1$.

La segunda, la refinería de Zinc en Cajamarquilla, inicialmente administrada por el Estado, a través de Minero Perú, refina parte de los concentrados de zinc provenientes de productores de la mediana minería. Se puede afirmar que durante los años que el Estado operó esta refinería supo transferir de mejor forma las condiciones de mercado imperante en esos momentos, así como que los impuestos cobrados se ajustaron a una justa valorización de la producción de concentrados y los refinados resultantes.

Ambas refinerías administradas por intermedio de la empresa estatal, limitó la práctica de la subvaluación o la sobre valorización (transferencia de precios) del producto minero, sea este en concentrados o refinados, así como el desarrollo de un Know how propio sobre la comercialización minera metalúrgica, siendo obvio el valor agregado que se generaba por la refinación.

Las refinerías privatizadas a un valor muy discutible durante el primer gobierno del presidente Fujimori y hoy pertenecientes a las compañías Votorantim Metais y Southern Perú, respectivamente.

\section{INTENTANDO RESPUESTAS}

Las interrogantes planteadas anteriormente podrían tener más de una respuesta, entre las cuales podríamos encontrar:

"Que se carece del ahorro interno suficiente para realizar inversiones de tal envergadura". "Que las mayores operaciones mineras se encuentran en manos de in-

versionistas extranjeros, las cuales remiten a sus principales del exterior las utilidades 


\section{Ysaac Ospino Edery}

obtenidas". "Que los actores de nuestra mediana minería -que es la que exporta la mayor cantidad de concentrados- no tendrían el mayor interés de exportar refinados ya que se podrían evidenciar los verdaderos valores de los concentrados". Otra, "Que se verían fuertemente afectados los intereses de las navieras, que manejan el negocio del flete marítimo, al disminuir los volúmenes por la exportación de refinados", ya que estos representan alrededor del $50 \%$ del peso que dejaría de fletarse; y por ultimo, que los gobiernos de turno adoptaron al pie de la letra las políticas liberales dejando de lado el actuar en un sector estratégico, situación contraria a la política de explotación y comercialización de nuestros vecinos del sur².

Tabla N. ${ }^{\circ}$ 1. Fórmulas y Variables

Fórmula $A: \quad V C=[M-D]\left[P . F-\left(R-+E^{n}\right)\right]-(T-+E 2)-X+Y$

Fórmula $\mathrm{B}: \quad \mathrm{VC}=[\mathrm{M}-\mathrm{D}][\mathrm{P} . \mathrm{F}]-[(\mathrm{T}-\mathrm{E})+\mathrm{T}(\mathrm{P}-\mathrm{PB})]-\mathrm{X}+\mathrm{Y}$

Donde:

VC: $\quad$ Valor del Concentrado.

M: $\quad$ Contenido de Metal.

D: $\quad$ Deducciones por perdida metalúrgica.

P: $\quad$ Precio del metal (cotización referencial utilizada).

F: $\quad$ Factor del Precio.

R: $\quad$ Maquila de Refinación (Refining Charge).

E1: $\quad$ Escalador de Maquila de Refinación.

T: $\quad$ Maquila de Tratamiento.

E2: $\quad$ Escalador de Maquila de Tratamiento.

PB: $\quad$ Precio Base referente al costo de tratamiento (Fundición y/o Refinación)

$\mathrm{T}: \quad$ Factor de Ajuste del Costo de Tratamiento.

$\mathrm{X}$ : Deducciones por impurezas.

Y: $\quad$ Créditos por Subproductos.

Fuente: Economía Minera - Alfredo Dammert Lira / Minpeco 1980/1990.

2. Decreto 76 de Minería. Política de Fomento para la Pequeña y Mediana Minería de Chile. "Para garantizar el acceso de la producción del sector a los mercados internacionales, en condiciones comerciales similares a las que obtienen los grandes productores que operan en Chile, la Empresa Nacional de Minería administrará poderes de compra de minerales, concentrados y precipitados, y proveerá servicios de beneficio de minerales. El valor a pagar por el producto recepcionado se calculará descontando al precio del metal los valores de mercado internacionales, de la fusión y refinación electrolítica de concentrados, así como también, en el caso de compra de minerales, los valores que simulen mercados eficientes, de transformación de minerales a concentrados o precipitados... Para la pequeña minería se cobrará el menor cargo de fusión y refinación que se haya acordado en los contratos regulares anuales de compra suscritos por la Empresa con la minería mediana e independiente". 


\section{Una aproximación a la comercialización de la producción de los concentrados mineros}

Según consultoras internacionales especializadas, la construcción y puesta en marcha de una refinería de cobre o zinc similares en capacidad a la de Ilo o Cajamarquilla podría fluctuar entre unos US\$1000 a US\$1200 millones de dólares cada una de ellas, a precios actuales.

"Una de las cuales podría haberse financiado, solo con el ahorro del flete en exceso pagado por la exportación de un concentrado con una ley promedio del $50 \%$ de fino pagable, dado que el $50 \%$ restante en la mayoría de los casos es material sin mayor valor comercial en la mayoría de los casos".

Veamos, entre 1989 y 2007 se han exportado alrededor de 23`000,000 TMS de concentrados de Zinc (no finas). Si se asume que un 55\% es fino, nos queda 10`350,000 de material sin mayor valor comercial que exportado (embarcado) a un costo estimado de 100 dólares el flete marítimo por TM tendríamos el monto de US\$1,035`000,000 millones de dólares, pagados por las compañías mineras al exportar sus concentrados a las empresas navieras de bandera extranjera. Aquí una conclusión obvia de quiénes ganan y quiénes pierden con esta situación.

Similar cálculo se realiza para las exportaciones de concentrado de cobre o plomo.

Con el ahorro en flete, se hubiera podido financiar una buena parte de una refinería similar a la que hoy opera en Cajamarquilla o Ilo.

Por otro lado, de la tabla $\mathrm{N} .^{\circ} 1$, la variable $\mathrm{R}$ (maquila de refinación), estimada en 100 dólares por TM, la que multiplicada por los volúmenes que se refinan (todos los concentrados que se exportan para poder tener acceso a los metales contenidos para su uso industrial, tienen que ser fundidos y refinados), se llegaría también a un monto impresionante cercano o superior a los 1,000`000,000 millones de dólares. Otra conclusión de quiénes ganan y quienes pierden.

El resto de valores de las variables de la tabla $\mathrm{N} .^{\circ} 1$ pueden ser ajustados (manipulados), a fin de evadir el pago de los impuestos correspondientes.

Un ejemplo teórico:

a) El vendedor $V$ acuerda la venta de la cantidad $Q$ de concentrados al comprador $C$. 


\section{Ysaac Ospino Edery}

b) El vendedor $V$ propone al comprador $Q$ una deducción, mayor de lo que fija el mercado en ese momento (estos podrían ser contenidos de impurezas por pérdida metalúrgica, contenido del metal, etc.), indicando (poniéndose de acuerdo) que el producto de la diferencia, sea depositada en una Cta. bancaria xxxx en un banco en las Bahamas, a nombre del vendedor.

c) No hay ninguna institución del Estado peruano que fiscalice o controle esta operación.

d) El vendedor logra evadir el pago del impuesto correspondiente, evidenciándose una vez más una operación de subvaluación o sobre valorización.

"No se pretende señalar que alguna empresa minera en particular esté realizando una forma de elusión tributaria, sino que dado el vacío de control - fiscalización por parte del Estado, es factible su práctica".

"La comercialización de los metales en el ámbito mundial se da en dos mercados que forman parte de una misma cadena que funciona de manera distinta. Por un lado, existe un mercado donde interactúan las empresas operadoras de minas que producen concentrados (ofertantes), y las refinerías de dichos concentrados (demandantes), que en número no supera al centenar para el caso del zinc.

Por otro lado, existe un mercado en el que interactúan las refinadoras como ofertantes y las empresas industriales como demandantes, las cuales se las cuentan por miles.

El primer mercado se caracteriza por que las empresas productoras no se encuentran integradas verticalmente (no cuentan con refinerías) y están alejadas de los demandantes industriales de refinados, lo que las obliga a vender sus concentrados a las empresas refinadoras. En este mercado, el precio que se negocia principalmente es el costo de la (maquila). El costo de la maquila se fija según las características del concentrado (contenido del metal principal, metales secundarios, impurezas).

Asimismo los contratos de maquila, incluyen un factor denominado (escalador), el cual disminuye el costo si la cotización del metal cae y lo eleva si esta última sube. El escalador hace que los ingresos de las refinerías sean sensibles a cambios en el precio del metal, trasladando los desequilibrios a sus abastecedores de concentrados. 


\section{Una aproximación a la comercialización de la producción de los concentrados mineros}

En el segundo mercado, el del metal refinado (metálico), se transan calidades cercanas al $99.99 \%$ de contenido fino, en este caso los ofertantes son las refinerías mientras que los compradores son las empresas industriales" 3 .

Cuadro N. ${ }^{\circ}$ 4. Minas y Refinerías de Zinc

\begin{tabular}{lrc}
\hline \multicolumn{1}{c}{ Ubicación } & Minas & Refinerías \\
\hline África & 6 & 3 \\
Asia & 19 & 46 \\
Australia & 9 & 3 \\
Latinoamérica & 64 & 7 \\
Norteamérica & 15 & 9 \\
Europa & 12 & 28 \\
Total & 125 & 96 \\
\hline
\end{tabular}

- Las más representativas

Fuente: ILZSG

El cuadro $\mathrm{N}^{\circ} 4$ nos presenta un balance de ofertantes y de demandantes de concentrados. En el caso de la oferta, el Perú en exportaciones de concentrados de zinc ocupa entre un tercer o cuarto puesto a escala mundial y dado que no cuenta con las refinerías suficientes para procesar (refinar), el volumen de concentrados que produce, se coloca en una posición de dependencia frente a las refinerías. Son las refinerías las que logran, con su posición en el mercado, definir las condiciones de refinación afectando la valorización de los concentrados que adquieren.

Veamos algunos términos que se consideran en una compra de concentrados de cobre, según Anexo N. ${ }^{\circ}$, las mismas que son susceptibles de manipulación.

\section{A MANERA DE CONCLUSIÓN}

El Estado peruano no tiene mayor control sobre el real valor de las exportaciones de concentrados, con lo cual posibilita la práctica de la sobre valorización y sub valuación (transferencia de precios) por lo cual urge la conformación de alguna forma de organización estatal que perfeccione dicho control.

3. Milla Bastas, Omar. Pacific Credit Rating: wwwratingspcr.com 


\section{Ysaac Ospino Edery}

Los montos acumulados, descontados en la valorización de concentrados por concepto de cargos por refinación, sugieren que se podría haber financiado una refinería en el periodo de análisis.

El actual modelo de promoción a la inversión minera no permite un proceso de acumulación real de ahorro interno, dado que la mayor parte de la inversión en minería obedece y depende de capitales foráneos.

La explotación minera y su comercialización ha representado desde hace más de un siglo la principal actividad económica de departamentos -hoy regiones- que presentan los más bajos índices de pobreza. Hoy en día hay similar modelo de explotación y comercialización, lo que pronostica mantener la población en la misma postración económica y social.

Los montos acumulados por pago de flete marítimo de exportación de concentrados mineros, en que alrededor del $50 \%$ del peso no tiene mayor valor comercial, permite conjeturar que hubiera sido factible el financiar una refinería o parte de una, con el ahorro generable.

\section{BIBLIOGRAFÍA}

Banco Central de Reserva del Perú. Memoria Anual del BCR: 2000- 2006.

Ministerio de Energía y Minas. Anuarios Mineros: 1985/2006.

Dammert Lira, Alfredo. "Economía Minera”. Minero Perú Comercial, 1990.

Asociación Latinoamericana de Zinc - LATIZA. Estadísticas: 1990/2006.

General Customs Administration PRC. Estadísticas: 1990/2006.

Mondragon, Margarita D. "Conferencia Taller Mercado de Zinc Refinado" Universidad Nacional de Ingeniería. Mayo de 2005.

Alcayaga O., Julian. "Comité de Defensa y Recuperación del Cobre de Chile".

Ospino Edery, Juan José. "La incidencia del Mercado Mundial en la Producción Nacional de Cobre". Tesis Facultad de Economía, UNMSM. 


\section{Anexo N. ${ }^{\circ} 1$}

\section{CONTRATO DE VENTA DE CONCENTRADOS DE COBRE ENTRE XXX S.R.L y XXX S.A}

1. Producto: Concentrado de Cobre de la calidad xxxxx con los siguientes ensayes típicos:

$\begin{array}{llll}\mathrm{Cu} \% & 21-29 & \mathrm{Bi} \% & 0.2-0.3 \\ \mathrm{Au} \mathrm{gr} / \mathrm{tm} & 0.5-0.7 & \mathrm{Hg} \mathrm{ppm} & 10-13.5 \\ \mathrm{As} \% & 4-8 & \mathrm{Sb} \% & 3-5 \\ \mathrm{~Pb} \% & 6-10 & \mathrm{Cd} \% & 0.1 \\ \mathrm{H}_{2} \mathrm{O} \% & 6.5-8.8 & \mathrm{Fe} & 8.6-11.7 \\ \mathrm{Zn} \% & 8-13 & \mathrm{Co} \mathrm{ppm} & 10 \\ \mathrm{~S} \% & 25.6-34.6 & & \\ \mathrm{Ins} & 4-6 & & \end{array}$

El comprador se reserva el derecho de rechazar cualquier entrega del producto cuyos contenidos no estén de acuerdo con los indicados en la presente cláusula, sin tener por esto ninguna responsabilidad.

2. Periodo (fechas de entrega).

3. Cantidad (volúmenes).

4. Entrega (lugar de entregas de acuerdo a INCOTERMS).

5. Precio.

El precio por tonelada métrica seca del producto será el resultado de la suma de los pagos menos las deducciones, como se especifica a continuación:

\subsection{Pagos por Contenidos Metálicos}

5.1.1 Cobre: $100 \%$ del contenido final de cobre en el Producto, previa deducción 1.1 unidades, al promedio de la cotización LME Grade "A" Settlement para el cobre, como se publica en la Revista Metals Week, para el periodo de cotización aplicable.

5.1.2 Plata: 95\% del contenido final de plata en el Producto, sujeto a una deducción mínima de 30 gr por tonelada métrica seca, al promedio de cotización London Spot para la plata, tal como se publica en al Revista Metals Week, para el periodo de cotización aplicable. 


\section{Ysaac Ospino Edery}

5.1.3 Oro: 95\% del contenido final de oro en el Producto.

5.2 Deducciones

5.2.1 Gastos de tratamiento: US\$ 105 (ciento cinco y 00/100 dólares americanos) por tonelada métrica seca del Producto, a un precio de base de US $\$ 0.90 / 1 \mathrm{~b}$ para el cobre refinado. Se incrementará el gasto de tratamiento en $10 \%$ por libra pagable de cobre por cada US $\$ 0.01 / 1 \mathrm{~b}$, en que el precio del cobre aplicable exceda al precio base de US $\$ 0.90 / \mathrm{lb}$.

5.2.2 Gasto de refinación por el Cobre: US $\$ 0.075$ por libra pagable de cobre en el Producto. Las fracciones se prorratearan.

5.2.3 Gastos de refinación por la Plata: US\$ 0.30 por onza troy pagable de plata en el Producto. Las fracciones se prorratearán.

5.2.4 Gasto de refinación por el Oro: US $\$ 8.50$ por onza troy pagable de oro en el Producto. Las fracciones se prorratearán.

5.2.5 Penalidad por Humedad: $10 \%$ libre, y US $\$ 3.00$ por cada $1.00 \%$ que el contenido final de Agua exceda $10 \%$.

5.2.6 Penalidad por Arsénico (As): $0.80 \%$ libre, y US\$ 1.50 por cada $0.10 \%$ que el contenido final de Arsénico exceda $0.80 \%$.

5.2.7 Penalidad por Antimonio (Sb): $0.60 \%$ libre, y US $\$ 2.00$ por cada $0.10 \%$ que el contenido final exceda $0.60 \%$.

5.2.8 Penalidad por Bismuto (Bi).

5.2.9 Penalidad por Plomo y Zinc.

5.2.10Penalidad por Alumina (AlO)

5.2.11Penalidad por Mercurio (Hg).

6. Periodo de Cotizaciones.

7. Pagos.

8. Determinacion de peso entregado, muestreo, determinación de humedad y determinación de ensayos.

9. Título y Riesgo.

10. Tributos. 